MATHEMATICS OF COMPUTATION

Volume 66, Number 219, July 1997, Pages 1341-1346

S 0025-5718(97)00870-3

\title{
ON WENDT'S DETERMINANT
}

\author{
CHARLES HELOU
}

\begin{abstract}
Wendt's determinant of order $m$ is the circulant determinant $W_{m}$ whose $(i, j)$-th entry is the binomial coefficient $\left(\begin{array}{c}m \\ |i-j|\end{array}\right)$, for $1 \leq i, j \leq m$. We give a formula for $W_{m}$, when $m$ is even not divisible by 6 , in terms of the discriminant of a polynomial $T_{m+1}$, with rational coefficients, associated to $(X+1)^{m+1}-X^{m+1}-1$. In particular, when $m=p-1$ where $p$ is a prime $\equiv-1(\bmod 6)$, this yields a factorization of $W_{p-1}$ involving a Fermat quotient, a power of $p$ and the 6 -th power of an integer.
\end{abstract}

\section{INTRODUCTION}

E. Wendt ([12]) introduced the $m \times m$ circulant determinant $W_{m}$ with first row the binomial coefficients $\left(\begin{array}{c}m \\ 0\end{array}\right),\left(\begin{array}{c}m \\ 1\end{array}\right), \ldots,\left(\begin{array}{c}m \\ m-1\end{array}\right)$, i.e.

$$
W_{m}=\left|\begin{array}{ccccc}
1 & \left(\begin{array}{c}
m \\
1
\end{array}\right) & \left(\begin{array}{c}
m \\
2
\end{array}\right) & \ldots & \left(\begin{array}{c}
m \\
m-1
\end{array}\right) \\
\left(\begin{array}{c}
m \\
m-1
\end{array}\right) & 1 & \left(\begin{array}{c}
m \\
1
\end{array}\right) & \ldots & \left(\begin{array}{c}
m-2 \\
m-2
\end{array}\right) \\
\vdots & \vdots & \vdots & & \vdots \\
\left(\begin{array}{c}
m \\
1
\end{array}\right) & \left(\begin{array}{c}
m \\
2
\end{array}\right) & \left(\begin{array}{c}
m \\
3
\end{array}\right) & \ldots & 1
\end{array}\right|,
$$

which is the resultant of the polynomials $X^{m}-1$ and $(X+1)^{m}-1$, in connection with Fermat's last theorem ([10]). E. Lehmer ([9]) proved that $W_{m}=0$ if and only if $m \equiv 0(\bmod 6)$, and that if $p$ is an odd prime number, then $W_{p-1}$ is divisible by $p^{p-2} q_{p}(2)$, where $q_{p}(2)=\frac{2^{p-1}-1}{p}$ is a Fermat quotient. L. Carlitz ([2]) determined $W_{p-1}$ modulo $p^{p-1}$, which he then used to find high powers of $p$ dividing $W_{p-1}$ in an application in the same connection ([3]). Factorizations of the integers $W_{m}$ for $m \leq 50$ were given in ([7]). The size of $W_{m}$ was investigated in ([1]). Granville and Fee ([5]) determined the prime factors of $W_{m}$ for all even $m \leq 200$ and consequently improved on a classical result about Fermat's equation. This was further improved in ([6]), where similar computations were carried up to $m \leq 500$.

In this article, we show that for all positive even integers $m$ not divisible by 6 ,

$$
W_{m}=-9^{h_{m}}\left(2^{m}-1\right)^{3}(m+1)^{m-4\left|h_{m}\right|} D_{m}^{6},
$$

where $D_{m}$ is the discriminant of a polynomial with rational coefficients whose roots are given by a rational function of those of $(X+1)^{m+1}-X^{m+1}-1$, and $h_{m}=2$ or -1 according as $m \equiv 2$ or $4(\bmod 6)$ respectively. In particular, if $p$ is a prime $\equiv-1(\bmod 6)$ then $D_{p-1}$ is a rational integer and we have the factorization

$$
W_{p-1}=-\frac{1}{9} q_{p}(2)^{3} p^{p-2} D_{p-1}^{6} .
$$

Received by the editor May 6, 1996.

1991 Mathematics Subject Classification. Primary 11C20; Secondary 11Y40, 11D41, 12E10.

(c)1997 American Mathematical Society 


\section{Preliminary Results}

For any positive integer $m$, let $\zeta_{m}$ be a primitive $m$-th root of unity in $\mathbb{C}$. By a well-known expression for circulant determinants ([12]),

$$
W_{m}=\prod_{j=0}^{m-1}\left(\sum_{k=0}^{m-1}\left(\begin{array}{c}
m \\
k
\end{array}\right) \zeta_{m}^{j k}\right)=\prod_{j=0}^{m-1}\left(\left(1+\zeta_{m}^{j}\right)^{m}-1\right) .
$$

Denote by $n$ an odd integer $\geq 3$ and consider the polynomial

$$
P_{n}(X)=(X+1)^{n}-X^{n}-1 .
$$

Its relation to Wendt's determinant is the following

Proposition 1. For any odd integer $n \geq 3$, the discriminant of $P_{n}$ is

$$
D\left(P_{n}\right)=(-1)^{\frac{n-1}{2}} n^{n-2} W_{n-1} .
$$

Proof. Since $P_{n}$ has degree $n-1$ and leading coefficient $n$, we have ([4] or [11]) $D\left(P_{n}\right)=(-1)^{\frac{(n-1)(n-2)}{2}} n^{-1} R\left(P_{n}, P_{n}^{\prime}\right)$, where $R\left(P_{n}, P_{n}^{\prime}\right)$ is the resultant of $P_{n}$ and its derivative $P_{n}^{\prime}$. We also have $R\left(P_{n}, P_{n}^{\prime}\right)=(n(n-1))^{n-1} \prod_{k=1}^{n-2} P_{n}\left(y_{k}\right)$, where $y_{k}=\frac{1}{\zeta_{n-1}^{k}-1}(1 \leq k \leq n-2)$ are the roots of $P_{n}^{\prime}(X)=n\left((X+1)^{n-1}-X^{n-1}\right)$ in C. Every $P_{n}\left(y_{k}\right)=\frac{1-\left(\zeta_{n-1}^{k}-1\right)^{n-1}}{\left(\zeta_{n-1}^{k}-1\right)^{n-1}}$, for $1 \leq k \leq n-2$. The product $\prod_{k=1}^{n-2}\left(1-\zeta_{n-1}^{k}\right)$ is the value at 1 of $\left(X^{n-1}-1\right) /(X-1)$, which is $n-1$. Moreover, since $n$ is odd,

$$
\prod_{k=1}^{n-2}\left(1-\left(\zeta_{n-1}^{k}-1\right)^{n-1}\right)=\prod_{k=0}^{n-2}\left(\left(1+\zeta_{n-1}^{k+\frac{n-1}{2}}\right)^{n-1}-1\right)=W_{n-1},
$$

by (1). Hence $\prod_{k=1}^{n-2} P_{n}\left(y_{k}\right)=\frac{W_{n-1}}{(n-1)^{n-1}}$ and the result follows by substitution.

Now the polynomial $P_{n}$ can be written $([8])$

$$
P_{n}(X)=X(X+1)\left(X^{2}+X+1\right)^{e_{n}} F_{n}(X),
$$

where $F_{n}$ lies in $\mathbb{Z}[X]$, is prime to $X(X+1)\left(X^{2}+X+1\right)$, has degree $d_{n}=n-3-2 e_{n}$ and leading coefficient $n$, with $e_{n}=0,1$ or 2 according as $n \equiv 0,2$ or $1(\bmod 3)$ respectively. It follows from (2) and (3) that $F_{n}(-X-1)=F_{n}(X)$ and $F_{n}(1 / X)=$ $F_{n}(X) / X^{d_{n}}$. Hence the set of roots $z$ of $F_{n}$ in $\mathbb{C}$ is partitioned into $r_{n}=d_{n} / 6$ orbits of 6 elements each, namely

$$
\operatorname{Orb}(z)=\left\{z, \frac{1}{z},-z-1,-\frac{1}{z+1},-\frac{z+1}{z},-\frac{z}{z+1}\right\} .
$$

Let $z_{1}, \ldots, z_{r_{n}}$ be representatives of the different orbits of roots of $F_{n}$. For every $1 \leq j \leq r_{n}$, let $g_{j}$ be the monic polynomial whose roots are the elements of $\operatorname{Orb}\left(z_{j}\right)$. A straightforward computation gives

$$
g_{j}(X)=X^{6}+3 X^{5}+t_{j} X^{4}+\left(2 t_{j}-5\right) X^{3}+t_{j} X^{2}+3 X+1 \quad\left(1 \leq j \leq r_{n}\right)
$$

where

$$
t_{j}=6-J\left(z_{j}\right), \quad J(X)=\frac{\left(X^{2}+X+1\right)^{3}}{X^{2}(X+1)^{2}}
$$

and

$$
F_{n}=n \prod_{j=1}^{r_{n}} g_{j}
$$


Moreover

$$
g_{j}(X)=X^{2}(X+1)^{2}\left(J(X)-J\left(z_{j}\right)\right) \quad\left(1 \leq j \leq r_{n}\right) .
$$

We now introduce the polynomial

$$
T_{n}(X)=\prod_{j=1}^{r_{n}}\left(X-t_{j}\right)
$$

which lies in $\mathbb{Q}[X]$, since the automorphisms of the splitting field of $F_{n}$ over $\mathbb{Q}$ permute the roots of $T_{n}$ and thus leave its coefficients fixed. Substituting (8) into (7) yields

$$
F_{n}(X)=(-1)^{r_{n}} n X^{2 r_{n}}(X+1)^{2 r_{n}} T_{n}(6-J(X)) .
$$

This relation, linking $T_{n}$ to $F_{n}$ and thus to $P_{n}$, facilitates computations with $T_{n}$.

\section{Discriminants CAlCUlations}

The resultant of two non-zero polynomials $f, g \in \mathbb{C}[X]$ is denoted by $R(f, g)$ and the discriminant of $f$ by $D(f)$. The classic formula $([4]) D(f g)=D(f) D(g) R(f, g)^{2}$ yields by induction

Lemma 1. If $f_{1}, \ldots, f_{m}$ are non-constant polynomials in $\mathbb{C}[X]$, then

$$
D\left(\prod_{i=1}^{m} f_{i}\right)=\prod_{i=1}^{m} D\left(f_{i}\right) . \prod_{1 \leq i<j \leq m} R\left(f_{i}, f_{j}\right)^{2} .
$$

Using this, the relation (3) allows, when $e_{n}<2$, to express $D\left(F_{n}\right)$ in terms of $D\left(P_{n}\right)$. Indeed,

Lemma 2. For a positive odd integer $n \not \equiv 1(\bmod 6)$,

$$
D\left(F_{n}\right)=\frac{(-1)^{e_{n}} D\left(P_{n}\right)}{3^{e_{n}} n^{4\left(e_{n}+1\right)}}
$$

Proof. Assume first $n \equiv-1(\bmod 6)$, so that $e_{n}=1$ and

$$
P_{n}(X)=X(X+1)\left(X^{2}+X+1\right) F_{n}(X) \text {. }
$$

From Lemma 1,

$$
D\left(P_{n}\right)=-3\left(F_{n}(0) F_{n}(-1) F_{n}\left(\zeta_{3}\right) F_{n}\left(\zeta_{3}^{2}\right)\right)^{2} D\left(F_{n}\right) .
$$

Now, for all odd $n, F_{n}(0)=F_{n}(-1)=n$, since these are the values of $P_{n}(X) / X$ at 0 and $-P_{n}(X) /(X+1)$ at -1 respectively. On the other hand, setting $P_{n}(X)=$ $\left(X^{2}+X+1\right) Q_{n}(X)$, with $Q_{n} \in \mathbb{Z}[X]$, we have

$$
F_{n}\left(\zeta_{3}\right)=\frac{Q_{n}\left(\zeta_{3}\right)}{\zeta_{3}\left(\zeta_{3}+1\right)}=-\frac{P_{n}^{\prime}\left(\zeta_{3}\right)}{2 \zeta_{3}+1}=-\frac{n\left(\left(\zeta_{3}+1\right)^{n-1}-\zeta_{3}^{n-1}\right)}{2 \zeta_{3}+1}=n .
$$

Also, $F_{n}\left(\zeta_{3}^{2}\right)$, being the complex conjugate of $F_{n}\left(\zeta_{3}\right)$, is equal to $n$ too. Hence $D\left(P_{n}\right)=-3 n^{8} D\left(F_{n}\right)$. Similarly, in the simpler case where $n \equiv 3(\bmod 6)$, we have $P_{n}(X)=X(X+1) F_{n}(X)$ so that $D\left(P_{n}\right)=\left(F_{n}(0) F_{n}(-1)\right)^{2} D\left(F_{n}\right)=n^{4} D\left(F_{n}\right)$.

We now relate the discriminants of $F_{n}, T_{n}$ and the $g_{j}$ 's.

Lemma 3. For any odd integer $n \geq 3$,

$$
D\left(F_{n}\right)=n^{2\left(d_{n}-1\right)} \cdot \prod_{j=1}^{r_{n}} D\left(g_{j}\right) \cdot D\left(T_{n}\right)^{6} .
$$


Proof. By (7) and Lemma 1, D(Fn $)=n^{2\left(d_{n}-1\right)} \cdot \prod_{j=1}^{r_{n}} D\left(g_{j}\right) \cdot \prod_{1 \leq i<j \leq r_{n}} R\left(g_{i}, g_{j}\right)^{2}$. By (8), for $1 \leq i, j \leq r_{n}, R\left(g_{i}, g_{j}\right)=\prod_{z} g_{j}(z)=\left(J\left(z_{i}\right)-J\left(z_{j}\right)\right)^{6}\left(\prod_{z} z(z+1)\right)^{2}$, where the products are for $z$ ranging in $\operatorname{Orb}\left(z_{i}\right)$, in which case $J(z)=J\left(z_{i}\right)$ by (5) and (6). Moreover, $\prod_{z} z=g_{j}(0)=1$ and $\prod_{z}(z+1)=g_{j}(-1)=1$. Hence $R\left(g_{j}, g_{i}\right)=R\left(g_{i}, g_{j}\right)=\left(J\left(z_{i}\right)-J\left(z_{j}\right)\right)^{6}$. On the other hand, $D\left(T_{n}\right)=$ $(-1)^{r_{n}\left(r_{n}-1\right) / 2} \prod_{i \neq j}\left(t_{i}-t_{j}\right)= \pm \prod_{i \neq j}\left(J\left(z_{j}\right)-J\left(z_{i}\right)\right)$, where the products are for all $i, j \in\left\{1, \ldots, r_{n}\right\}$ with $i \neq j$. Hence $\prod_{1 \leq i<j \leq r_{n}} R\left(g_{i}, g_{j}\right)^{2}=\prod_{i \neq j} R\left(g_{i}, g_{j}\right)=$ $\prod_{i \neq j}\left(J\left(z_{i}\right)-J\left(z_{j}\right)\right)^{6}=D\left(T_{n}\right)^{6}$ and the result follows.

Next, we compute the dicriminants of the $g_{j}$ 's.

Lemma 4. For any odd integer $n \geq 3$ and $1 \leq j \leq r_{n}$,

$$
D\left(g_{j}\right)=-\left(4 t_{j}+3\right)^{3}\left(t_{j}-6\right)^{4} .
$$

Proof. Let $Y=X+1 / X$. Then $g_{j}(X)=X^{3} h_{j}(Y)$, where $h_{j}(Y)=Y^{3}+3 Y^{2}+$ $\left(t_{j}-3\right) Y+2 t_{j}-11$; and $g_{j}^{\prime}(X)=3 g_{j}(X) / X+\left(X^{3}-X\right) h_{j}^{\prime}(Y)$. Hence

$$
D\left(g_{j}\right)=-\prod_{z} g_{j}^{\prime}(z)=-\left(\prod_{z} z\right)\left(\prod_{z}(z+1)\right)\left(\prod_{z}(z-1)\right) \prod_{z} h_{j}^{\prime}\left(z+\frac{1}{z}\right),
$$

where the products are for $z \in \operatorname{Orb}\left(z_{j}\right)$. From the proof of Lemma $3, \prod_{z} z=$ $\prod_{z}(z+1)=1$. Also $\prod_{z}(z-1)=g_{j}(1)=4 t_{j}+3$. Moreover, $y=z+1 / z$ ranges through the roots of $h_{j}$, each repeated twice, as $z$ ranges through $\operatorname{Orb}\left(z_{j}\right)$, so that $\prod_{z} h_{j}^{\prime}(z+1 / z)=\left(\prod_{y} h_{j}^{\prime}(y)\right)^{2}=D\left(h_{j}\right)^{2}$. Thus $D\left(g_{j}\right)=-\left(4 t_{j}+3\right) D\left(h_{j}\right)^{2}$. Now, setting $U=Y+1$, we have $h_{j}(Y)=f_{j}(U)=U^{3}+\left(t_{j}-6\right) U+t_{j}-6$. By a well-known formula for the discriminant of a cubic polynomial ([11]), we get $D\left(h_{j}\right)=D\left(f_{j}\right)=-\left(4 t_{j}+3\right)\left(t_{j}-6\right)^{2}$. Hence the result.

The product, appearing in Lemma 3 , of the discriminants of the $g_{j}$ 's is given by

Lemma 5. For any odd integer $n \geq 3$,

$$
\prod_{j=1}^{r_{n}} D\left(g_{j}\right)=(-1)^{r_{n}} 3^{4-7 e_{n}}\left(2^{n-1}-1\right)^{3} n^{4 e_{n}-7}\left(\frac{n-1}{2 n}\right)^{2 e_{n}\left(e_{n}-1\right)} .
$$

Proof. By Lemma 4,

$$
\prod_{j=1}^{r_{n}} D\left(g_{j}\right)=(-1)^{r_{n}}\left(\prod_{j=1}^{r_{n}}\left(4 t_{j}+3\right)\right)^{3}\left(\prod_{j=1}^{r_{n}}\left(t_{j}-6\right)\right)^{4} .
$$

Now $\prod_{j=1}^{r_{n}}\left(4 t_{j}+3\right)=(-4)^{r_{n}} T_{n}(-3 / 4)$. Moreover, substituting $X=1$ into (10) and (3), we get $(-4)^{r_{n}} n T_{n}(-3 / 4)=F_{n}(1)=P_{n}(1) /\left(2.3^{e_{n}}\right)$. Hence

$$
\prod_{j=1}^{r_{n}}\left(4 t_{j}+3\right)=\frac{F_{n}(1)}{n}=\frac{2^{n-1}-1}{3^{e_{n}} n} .
$$

Similarly, $\prod_{j=1}^{r_{n}}\left(t_{j}-6\right)=(-1)^{r_{n}} T_{n}(6)$, and substituting $X=\zeta_{3}$ into (10) yields $(-1)^{r_{n}} n T_{n}(6)=F_{n}\left(\zeta_{3}\right)$. Let $Q_{n}(X)=X(X+1) F_{n}(X)$; then $F_{n}\left(\zeta_{3}\right)=-Q_{n}\left(\zeta_{3}\right)$ and, by (3), $P_{n}(X)=\left(X^{2}+X+1\right)^{e_{n}} Q_{n}(X)$. Taking $e_{n}$-th derivatives in the latter relation and making $X=\zeta_{3}$, we get $Q_{n}\left(\zeta_{3}\right)=P_{n}^{\left(e_{n}\right)}\left(\zeta_{3}\right) /\left(e_{n} !\left(2 \zeta_{3}+1\right)^{e_{n}}\right)$ (here 
$2 \zeta_{3}+1$ is the value of the factor $X-\zeta_{3}^{2}$ in $X^{2}+X+1$, and the equality follows from Taylor's formula). Hence

$$
\prod_{j=1}^{r_{n}}\left(t_{j}-6\right)=\frac{F_{n}\left(\zeta_{3}\right)}{n}=-\frac{P_{n}^{\left(e_{n}\right)}\left(\zeta_{3}\right)}{e_{n} ! n\left(2 \zeta_{3}+1\right)^{e_{n}}} .
$$

Simple computations show that $-P_{n}^{\left(e_{n}\right)}\left(\zeta_{3}\right) /\left(2 \zeta_{3}+1\right)^{e_{n}}=3$ or $n$ or $n(n-1) / 3$ according as $n \equiv 0$ or 2 or $1(\bmod 3)$ respectively. Therefore $\prod_{j=1}^{r_{n}}\left(t_{j}-6\right)=3 / n$ or 1 or $(n-1) / 6$ respectively. One formula representing all three cases is

$$
\prod_{j=1}^{r_{n}}\left(t_{j}-6\right)=\left(\frac{n}{3}\right)^{e_{n}-1}\left(\frac{n-1}{2 n}\right)^{\frac{e_{n}\left(e_{n}-1\right)}{2}} .
$$

Substituting (12) and (13) into (11) yields the desired result.

\section{Conclusion}

We can now draw the formula relating Wendt's determinant $W_{n-1}$ to the discriminant of the polynomial $T_{n}$, namely

Proposition 2. For any odd positive integer $n \not \equiv 1(\bmod 6)$,

$$
W_{n-1}=-9^{2-3 e_{n}}\left(2^{n-1}-1\right)^{3} n^{n+4 e_{n}-9} D\left(T_{n}\right)^{6},
$$

where $e_{n}=0$ or 1 according as $n \equiv 3$ or $-1(\bmod 6)$ respectively, and $T_{n}$ is defined by $(9)$.

Proof. By Lemmas 3 and 5 , since $d_{n}=n-3-2 e_{n}$ and $e_{n}=0$ or 1 , we have $D\left(F_{n}\right)=(-1)^{r_{n}} 3^{4-7 e_{n}}\left(2^{n-1}-1\right)^{3} n^{2 n-15} D\left(T_{n}\right)^{6}$. On the other hand, Proposition 1 and Lemma 2 imply $D\left(F_{n}\right)=(-1)^{e_{n}+(n-1) / 2} 3^{-e_{n}} n^{n-4 e_{n}-6} W_{n-1}$. Equating the two expressions (and noting that $r_{n}+e_{n}+(n-1) / 2=2\left(n+e_{n}\right) / 3-1$ is odd) yields the desired result.

Remark. In Proposition 2, let $m=n-1$ and $h_{m}=2-3 e_{n}$, so that $m$ is an even positive integer $\not \equiv 0(\bmod 6)$ and $h_{m}=2$ or -1 according as $m \equiv 2$ or $4(\bmod 6)$ respectively. Noting that $2-e_{n}$ coincides with $\left|h_{m}\right|$ and writing $D_{m}$ for $D\left(T_{m+1}\right)$, we obtain the formula for $W_{m}$ stated in the Introduction.

Assume now that $n=p$ is a prime number $\equiv-1(\bmod 6)$. Then the leading coefficient $p$ of $P_{p}$ divides all its coefficients $\left(\begin{array}{l}p \\ k\end{array}\right)$, for $1 \leq k \leq p-1$, so that, by (3), $F_{p}=p E_{p}$ where $E_{p}$ is a monic polynomial in $\mathbb{Z}[X]$. Thus the roots of $F_{p}$ are algebraic integers. Since, by (5), $t_{j}$ is a sum of products of roots of $F_{p}$, then $t_{j}$ is also an algebraic integer, for $1 \leq j \leq r_{p}$. Hence $T_{p}$ has rational integer coefficients and $D\left(T_{p}\right)$ lies in $\mathbb{Z}$. Therefore Proposition 2 (where now $e_{p}=1$ ) implies

Corollary. If $p$ is a prime number $\equiv-1(\bmod 6)$, then

$$
W_{p-1}=-\frac{1}{9} q_{p}(2)^{3} p^{p-2} D\left(T_{p}\right)^{6}
$$

where the discriminant $D\left(T_{p}\right)$ is a rational integer and $q_{p}(2)=\frac{2^{p-1}-1}{p}$. 


\section{REFERENCES}

1. D. Boyd, The asymptotic behaviour of the binomial circulant determinant, J. Math. Anal. Appl. 86 (1982), 30-38. MR 83f:10007

2. L. Carlitz, A determinant connected with Fermat's last theorem, Proc. Amer. Math. Soc. 10 (1959), 686-690. MR 21:7182

3. L. Carlitz, A determinant connected with Fermat's last theorem, Proc. Amer. Math. Soc. 11 (1960), 730-733. MR 22:7974

4. P. M. Cohn, Algebra, vol. 1, 2nd ed., J. Wiley and sons, New York, 1982. MR 83e:00002

5. G. Fee, A. Granville, The prime factors of Wendt's binomial circulant determinant, Math. Comp. 57 (1991), 839-848. MR 92f: 11183

6. D. Ford, V. Jha, On Wendt's determinant and Sophie Germain's theorem, Experimental Math. 2 (1993), 113-119. MR 95b:11029

7. J. S. Frame, Factors of the binomial circulant determinant, Fibonacci Quart. 18 (1980), 9-23. MR 81j: 10007

8. C. Helou, Cauchy's polynomials and Mirimanoff's conjecture, preprint.

9. E. Lehmer, On a resultant connected with Fermat's last theorem, Bull. Amer. Math. Soc. 41 (1935), 864-867.

10. P. Ribenboim, 13 Lectures on Fermat's last theorem, Springer, New York, 1979. MR 81f:10023

11. B. L. van der Waerden, Algebra, vol. 1, F. Ungar Pub. Co., New York, 1970. MR 41:8187a

12. E. Wendt, Arithmetische Studien über den letzten Fermatschen Satz, welcher aussagt, dass die Gleichung $a^{n}=b^{n}+c^{n}$ für $n>2$ in ganzen Zahlen nicht auflösbar ist, J. reine angew. Math. 113 (1894), 335-347.

Penn State University, Delaware County, 25 Yearsley Mill Road, Media, PennsylVANIA 19063

E-mail address: cxh22@psu.edu 